\title{
Regimes of reaction front propagation from ignition spots in $n$ - heptane/air mixtures laden with fine water mists
}

\author{
Zhou Yu ${ }^{1}$, Huangwei Zhang*1 \\ ${ }^{1}$ Department of Mechanical Engineering, National University of Singapore, Singapore \\ ${ }^{*}$ Corresponding author email: huangwei.zhang@nus.edu.sg
}

\begin{abstract}
The effects of water droplets on autoignition and detonation development induced by ignition spot with thermal inhomogeneity in $n-\mathrm{C}_{7} \mathrm{H}_{16}$ /air mixture are numerically investigated. The Eulerian-Eulerian method considering gas-liquid two-way coupling is applied. Three initial water droplet diameters are considered, i.e., 5, 10 and $20 \mu \mathrm{m}$. Zero-dimensional two-phase homogeneous ignitions under the constant-volume condition are studied first. Different ignition delay time and evaporation time scales are highlighted. It is seen that $\tau_{i g}$ of $5 \mu \mathrm{m}$ shows nonmonotonic change with $\alpha_{d, 0}$, indicating the NTC behaviours due to the water droplet addition. However, for 10 and $20 \mu \mathrm{m}$, increased $\alpha_{d, 0}$ leads to monotonically increased $\tau_{i g}$. Then, onedimensional two-phase autoignition and detonation development caused by temperature gradients in ignition spots is then discussed. It is found that conventional classification (i.e., (I) supersonic deflagrative wave, (II) detonative wave and (III) subsonic deflagrative wave) is also suitable for droplet laden mixtures. However, new regimes are observed for $5 \mu \mathrm{m}$.
\end{abstract}

\section{Keywords}

Autoignition; detonation; $n$-heptane; ignition spot; water droplets

\section{Introduction}

Downsizing of spark ignition engines (SIEs) with turbocharging technology has been deemed a megatrend since it can provide a novel solution for $\mathrm{CO}_{2}$ reduction and fuel economy [1]. However, knocking combustion may occur under some unfavourable conditions, and has become a stumbling block for SIEs with high compression ratio. It is known that knocking combustion is caused by end-gas autoignition and its formation is associated with the interactions between acoustic wave and chemical reaction [1]. In particular, autoignition and detonation development subject to localized reactivity non-uniformity (e.g. temperature inhomogeneity) in the chamber is believed to play a dominant role in inducing this peculiar phenomenon [2,3]. Therefore, how to suppress the engine knock arising from the non-uniform reactivity is an outstanding issue for performance improvement and technological innovation of SIEs.

Based on the pioneering work by Zel'dovich [4], different autoignition modes caused by a hot spot with thermal inhomogeneity are identified: i.e., subsonic reaction wave, detonation development and supersonic reaction wave. A detonation peninsula, parameterized by two non-dimensional parameters related to the hot spot (normalized temperature gradient $\xi$ and normalized acoustic time $\varepsilon$ ), is introduced by Bradley and his co-workers [5-8]. Grounded on the reactivity gradient theory, numerous simulations considering simplified or detailed chemical mechanisms have been performed to uncover the underpinning mechanism of autoignition and detonation development from a localized ignition spot. Dai et al. [9-13] investigate the various effects on autoignition and detonation development in DME/air and $n$ $\mathrm{C}_{7} \mathrm{H}_{16}$ /air mixtures under engine-relevant conditions. Both cold and hot spots are used to generate the autoignition reaction wave. It is known that, as an effective explosion inhibitor, water mist is widely used in preventing and reducing gas explosion hazard, particularly due to 
its easy availability [14,15]. Zhuang et al. [16] study hot spot induced autoignition and detonation characteristics of $n-\mathrm{C}_{7} \mathrm{H}_{16}$ /air mixture laden with liquid water droplets. The effects of droplet diameter and number density on reactive front development are discussed in their work. However, detonation development regime associated with properties of the hot spot in water-containing mixtures is not studied therein. Besides, the effects of water vapour dilution on autoignition and detonation development induced by ignition spot with thermal inhomogeneity in $n-\mathrm{C}_{7} \mathrm{H}_{16}$ /air mixture is numerically investigated in our previous work [17]. However, the effects of water mist remain to be explained.

In this study, detailed numerical simulations of autoignition and detonation development induced by temperature gradient in stoichiometric water-droplet laden $n-\mathrm{C}_{7} \mathrm{H}_{16}$ /air mixtures are conducted. The aims of our work are to answer the following questions: (1) how does water droplets (parameterised by initial droplet diameter $d_{0}$, droplet temperature $T_{d}$ as well as volume fraction $\alpha_{d}$ ) influence homogenous $n-\mathrm{C}_{7} \mathrm{H}_{16} /$ air autoignition? and (2) how does water droplets characterized by the corresponding parameters influence autoignition and detonation development from localized thermal non-uniformity?

\section{Governing Equation and Computational Method}

The governing equations of momentum, energy, and species mass fraction are solved for onedimensional unsteady, multi-component, reacting flows. They can be written in a spherical coordinate as

$$
\frac{\partial U}{\partial t}+\frac{\partial F(U)}{\partial r}+2 \frac{G(U)}{r}=F_{v}(U)+S_{R}+S_{L}
$$

where $t$ and $r$ are time and radial coordinate, respectively. The vectors $U, F(U), F_{v}(U), G(U)$, $S_{R}$ can be found in our previous work [17] and droplets induced source/sink terms $S_{L}$ are introduced in the following.

The Eulerian approach is applied to describe the liquid droplet phase. Similar approach is also used by Sanjosé et al. [18], Qiao et al. [19] for gas-liquid and gas-solid two-phase flows. In this study, the water droplet is assumed to be spherical. The droplet temperature is uniform due to the approximation of droplet infinite thermal conductivity, which equals the droplet surface temperature. The droplet breakup and deformation are not considered due to the small droplet diameters. The evolution of droplet diameter is governed by

$$
\frac{\partial d}{\partial t}+u_{d} \frac{\partial d}{\partial r}=-\frac{2 \dot{m}}{\pi \rho_{d} d^{2}}
$$

where $d, u_{d}, \dot{m}$ and $\rho_{d}$ are the droplet diameter, velocity, evaporation rate and material density, respectively.

The equation of droplet velocity takes the following form

$$
\frac{\partial u_{d}}{\partial t}+u_{d} \frac{\partial u_{d}}{\partial r}=\frac{F_{s}}{m_{d}}
$$

Note that only drag force $F_{S}$ is considered in our work and it is modelled using Schiller and Naumann's correlation [20]. $m_{d}=\rho_{d} \pi d^{3} / 6$ is the mass of a single droplet. $\tau_{r}$ is the droplet momentum relaxation time.

The equation of droplet temperature reads,

$$
m_{d} C_{P, d}\left(\frac{\partial T_{d}}{\partial t}+u_{d} \frac{\partial T_{d}}{\partial r}\right)=h_{c} A_{d}\left(T-T_{d}\right)-\dot{m} L_{v}\left(T_{d}\right)
$$


where $C_{P, d}$ is the constant pressure specific heat of the liquid phase and $A_{d}$ is the surface aera of a single droplet. $L_{v}\left(T_{d}\right)$ is the latent heat at the droplet temperature and approximated as [21]. $h_{c}$ is the convective heat transfer coefficient.

The equation of droplet number density $N_{d}$ reads,

$$
\frac{\partial N_{d}}{\partial t}+\frac{\partial\left(N_{d} u_{d}\right)}{\partial r}+2 \frac{N_{d} u_{d}}{r}=0
$$

In this study, two-way coupling between the gas and droplet phase is considered, characterized by the exchange of species, momentum and energy. They correspond to the individual terms in $S_{L}$ in Eq. (1) [18],

$$
\left\{\begin{array}{c}
S_{m}=N_{d} \dot{m} \\
S_{v}=-N_{d} m_{d} \frac{u-u_{d}}{\tau_{r}} \\
S_{e}=-N_{d} h_{c} A_{d}\left(T-T_{d}\right)+N_{d} \dot{m}_{d} H_{g}\left(T_{d}\right)
\end{array}\right.
$$

$S_{m}$ is non-zero only for the equation of $\mathrm{H}_{2} \mathrm{O}$ mass fraction. $H_{g}\left(T_{d}\right)$ is the enthalpy of water vapour at droplet temperature.

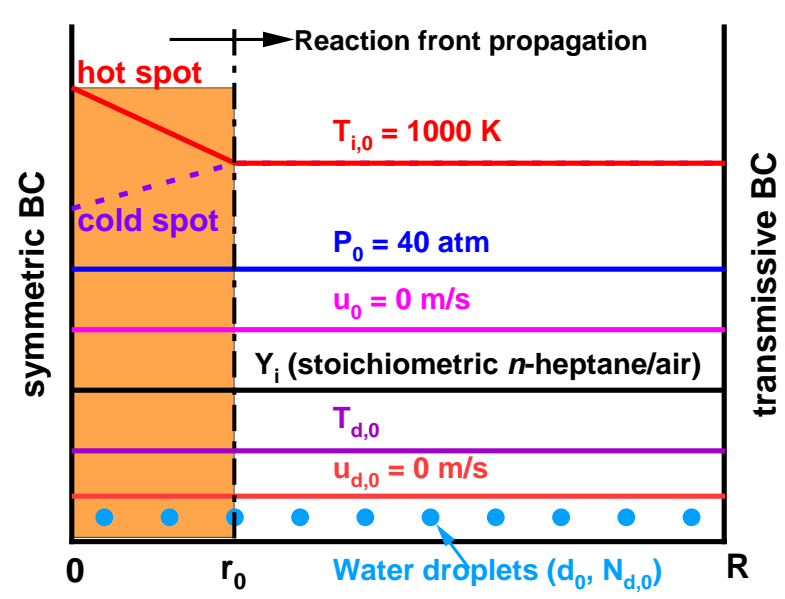

Figure 1. Schematic of the one-dimensional spherical semi-closed reactor.

One-dimensional spherical semi-closed reactor is studied in this work, which is schematically shown in Figure 1. The radius of the domain is $R=4 \mathrm{~cm}$. For the gas phase, the initial distributions can be found in our previous work. Fine water droplets are considered in this study to mimic the water mists injected into IC engine cylinder to mitigate the knock intensity and reduce pollutant emissions [1]. The water droplets are assumed to be mono-sized and three initial droplet diameters are considered, i.e., $d_{0}=5,10$, and $20 \mu \mathrm{m}$. The initial droplet temperature is $T_{d, 0}=298 \mathrm{~K}$. The initial droplet velocity is zero, i.e., $u_{d, 0}=0 \mathrm{~m} / \mathrm{s}$. In this study, the initial number density $N_{d, 0}$ is determined based on the initial droplet diameter $d_{0}$ and volume fraction $\alpha_{d, 0}$, i.e., $N_{d, 0}=\frac{\alpha_{d, 0}}{\pi d_{0}{ }^{3} / 6}$. In this study, dilute droplets are studied here and hence $\alpha_{d, 0} \leq 1.0 \times 10^{-3}$, as suggested by Crowe et al. [22]. Note that the effects of polydisperse are not considered, which may induce multi autoignition spots.

The autoigniting reactive front is initiated by a spot with temperature gradient near the left boundary, and therefore the initial temperature distribution $T_{0}$ in the reactor is 


$$
T_{0}(r)=T(t=0, r)=\left\{\begin{array}{rr}
T_{i, 0}+\left(r-r_{0}\right) \frac{d T_{0}}{d r}, & \text { for } 0 \leq r \leq r_{0} \\
T_{i, 0}, & \text { for } r_{0}<r \leq R_{W}
\end{array}\right.
$$

where $r_{0}$ is the radius of the ignition spot. $d T_{0} / d r$ is the initial temperature gradient inside the spot, which is constant and varied in our studies. $T_{i, 0}$ is the initial temperature beyond the spot, and is fixed to be $1,000 \mathrm{~K}$ in this study, close to the end gas temperature in IC engines [23,24]. The governing equations of gas and liquid phases are solved using a well-validated in-house code A-SURF (Adaptive Simulation of Unsteady Reactive Flow) $[25,26]$. This has been proven to be an accurate tool for predicting shock and detonation waves. The numerical details can be found in our previous work [17].

\section{Results and Discussion}

\section{Homogeneous ignitions of two-phase mixtures}

To quantify the autoignition process in an ignition spot with temperature gradient, three parameters are used, i.e., ignition delay time $\tau_{i g}$, excitation time $\tau_{e}$, and critical temperature gradient $(d T / d r)_{c}[6]$. Specifically, $\tau_{i g}$ is the duration when the heat release rate reaches its maximum from the initial instant, whilst $\tau_{e}$ denotes the time interval from $5 \%$ to maximum heat release [6]. Moreover, based on the theories by Zel'dovich [4] and Gu et al. [6], $(d T / d r)_{c}$ quantifies a critical temperature gradient within the ignition spot for chemical resonance and hence detonation development, i.e.,

$$
\left(\frac{d T}{d r}\right)_{c}=\left[a\left(\frac{d \tau_{i g}}{d T_{0}}\right)\right]^{-1}
$$

where $a=\sqrt{k R_{g} T}$ is the sound speed. $k$ is the adiabatic index and $R_{g}$ is the gas constant. Homogeneous ignitions of stoichiometric $n-\mathrm{C}_{7} \mathrm{H}_{16} /$ air mixtures with fine water droplets in the 1D reactor in Figure 1 are discussed in the following, to evaluate the effects of the droplet diameter and volume fraction on the abovementioned parameters, i.e., $\tau_{i g}, \tau_{e}$, and $(d T / d r)_{c}$. Here all initial variables of gas and liquid phases are spatially uniform, thereby leading to nominally homogeneous zero-dimensional simulations.

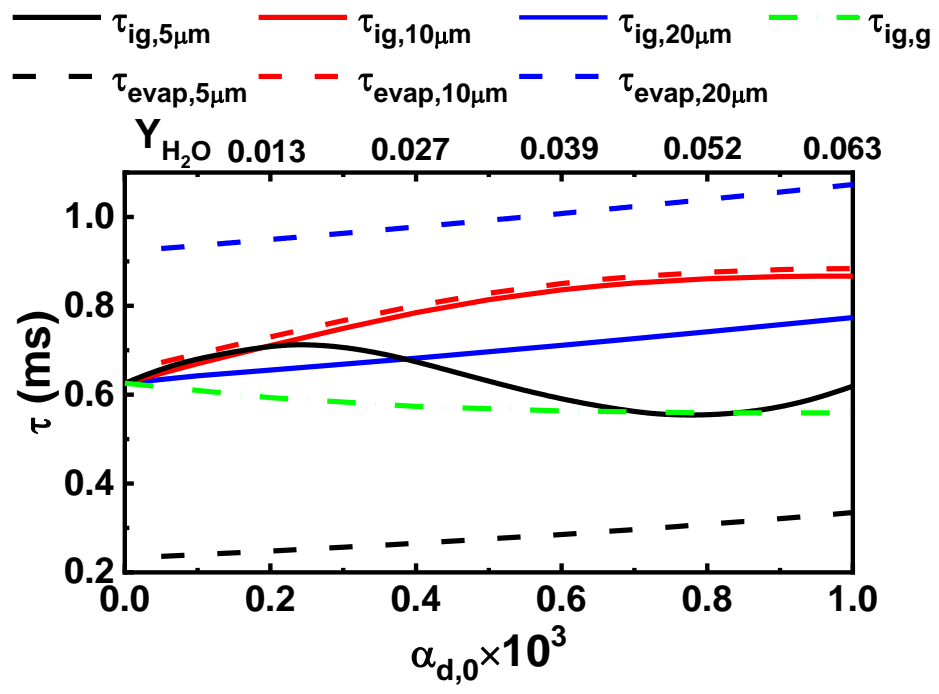

Figure 2. Ignition delay time and droplet evaporation time as functions of initial volume fraction. $T_{0}=1,000 \mathrm{~K}$. $Y_{\mathrm{H}_{2} \mathrm{O}}$ along the top axis indicates the water vapour mass fraction when the droplets are fully vaporized. 
Figure 2 shows the ignition delay time, droplet evaporation time, with different initial volume fractions $\alpha_{d, 0}$. The droplet evaporation time $\tau_{\text {evap }}$ is the instant when the droplet diameters in the reactor are reduced to $d \leq 10^{-12} \mu \mathrm{m}$. To evaluate the water vapour dilution effects, fully pre-vaporized results $\tau_{i g, g}$ (fully vaporized droplets in stoichiometric $n-\mathrm{C}_{7} \mathrm{H}_{16} /$ air mixture, $T_{0}=$ $1,000 \mathrm{~K})$ are also added. It is seen that $\tau_{i g, g}$ decreases slightly with nominal $\alpha_{d, 0}$, due to strengthened $\mathrm{R} 15\left(2 \mathrm{OH}+\mathrm{M}<=>\mathrm{H}_{2} \mathrm{O}_{2}+\mathrm{M}\right)$ [17]. Besides, $\tau_{i g}$ of $5 \mu \mathrm{m}$ shows non-monotonic change with $\alpha_{d, 0}$, indicating the NTC behaviours due to the water droplet addition. However, for 10 and $20 \mu \mathrm{m}$, increased $\alpha_{d, 0}$ leads to monotonically increased $\tau_{i g}$. Besides, $\tau_{\text {evap }}$ of $5 \mu \mathrm{m}$ is much smaller than the corresponding $\tau_{i g}$ for all the shown range of $\alpha_{d, 0}$. This indicates that ignition of $5 \mu \mathrm{m}$ proceeds in purely gaseous mixtures. However, $\tau_{\text {evap }}$ of $10 \mu \mathrm{m}$ is slightly larger than the corresponding $\tau_{i g}$. For $20 \mu \mathrm{m}, \tau_{\text {evap }}$ increases linearly with $\alpha_{d, 0}$ and is obviously larger than the corresponding $\tau_{i g}$.

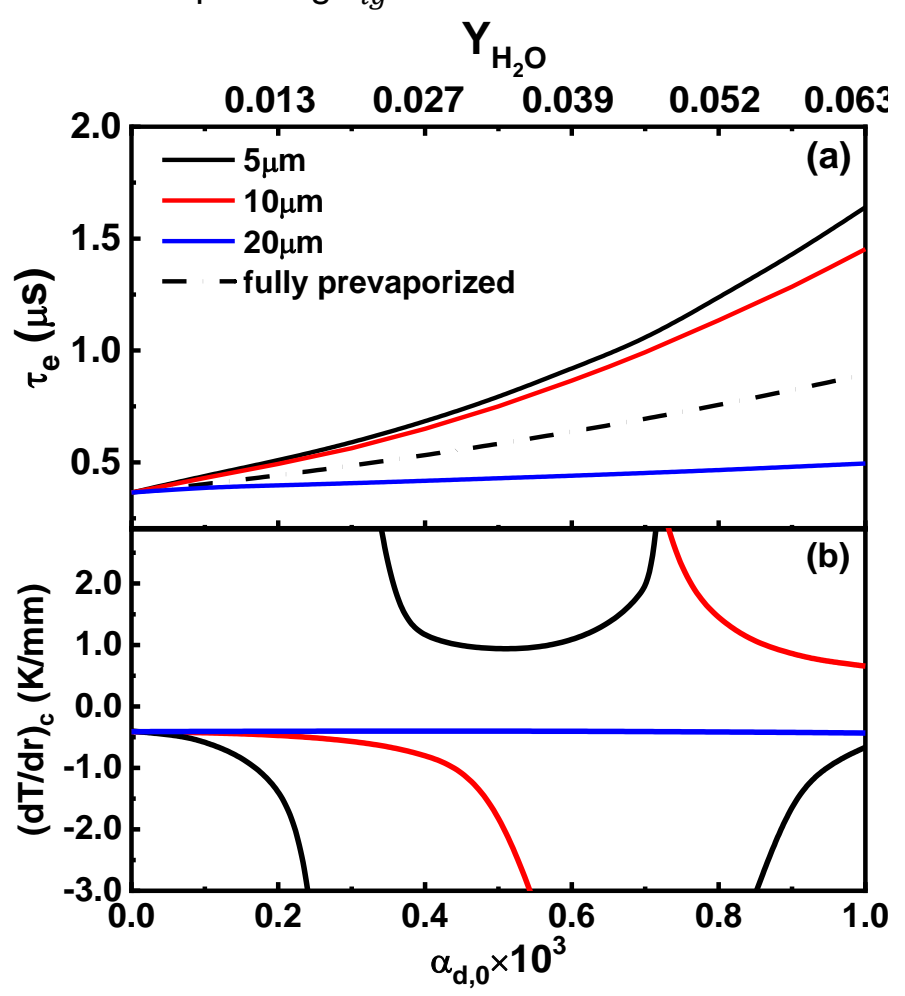

Figure 3. (a) Excitation time and (b) critical temperature gradient as functions of initial volume fraction. $T_{0}=1,000$ K. $Y_{\mathrm{H}_{2} \mathrm{O}}$ along the top axis indicates the water vapour mass fraction when the droplets are fully vaporized.

Figure 3 shows the excitation time $\tau_{e}$ and critical temperature gradient $(d T / d r)_{c}$ as functions of initial droplet volume fraction $\alpha_{d, 0}$. To evaluate the water vapour dilution effects, fully prevaporized results are also added. It is seen from Figure $3(\mathrm{a})$ that $\tau_{e}$ of all three $d_{0}$ increases monotonically with $\alpha_{d, 0}$. This is because the increased effects of cooling and dilution with more droplet evaporation. For a fixed $\alpha_{d, 0}, \tau_{e}$ becomes larger with smaller water droplets. For 5 and $10 \mu \mathrm{m}$, The difference of $\tau_{e}$ between droplet-laden mixtures and fully pre-vaporized mixtures is caused by the cooling effect. One can see that the cooling effect becomes more crucial with increased $\alpha_{d, 0}$. The change of critical temperature gradient $(d T / d r)_{c}$ as functions of initial droplet volume fraction $\alpha_{d, 0}$ is shown in Figure $3(\mathrm{~b})$. It is noted that a negative (positive) value of $(d T / d r)_{c}$ indicates that a hot (cold) spot is required for simulations of inhomogeneous 
mixture in following part. Due to the effect of NTC, there are three sections of the critical temperature for $5 \mu \mathrm{m}$ and two sections for $10 \mu \mathrm{m}$. For $20 \mu \mathrm{m},(d T / d r)_{c}$ keeps negative.

\section{Reaction front development from an ignition spot in two-phase mixtures}

Autoignition and detonation development due to the ignition spot with temperature gradient in water-droplet-laden $n-\mathrm{C}_{7} \mathrm{H}_{16}$ /air mixtures will be studied in this Section. Two parameters are used to characterize the interactions between the reaction wave and acoustic wave within the ignition spot $[5,6]$. The first one is the normalized temperature gradient $\xi$, which is the ratio of local sound speed to autoignition front propagation speed and measures the coupling between the local autoignition and acoustic wave caused by the heat release (or acoustic-induction coupling [27]). It reads,

$$
\xi=\frac{a_{r_{0} / 2}}{u_{a}}=\frac{d T_{0} / d r}{\left(d T_{0} / d r\right)_{c, r_{0} / 2}}
$$

where $d T_{0} / d r$ is the initial temperature gradient within the ignition spot (see Eq. 20), whereas $\left(d T_{0} / d r\right)_{c, r_{0} / 2}$ is the critical temperature gradient. The subscript " $r_{0} / 2$ " indicates the quantity is estimated based on the initial thermochemical properties in the middle of the ignition spot. Besides, the second parameter, $\varepsilon$, is used to measure the time scale of reaction heat release relative to the residence time of the acoustic wave in the ignition spot (or acousticexothermicity coupling). It is defined as the ratio of acoustic time to excitation time, i.e.,

$$
\varepsilon=\frac{r_{0} / a_{r_{0} / 2}}{\tau_{e}}
$$

Here $\tau_{e}$ is from OD calculations based on the properties at the middle of the ignition spot.

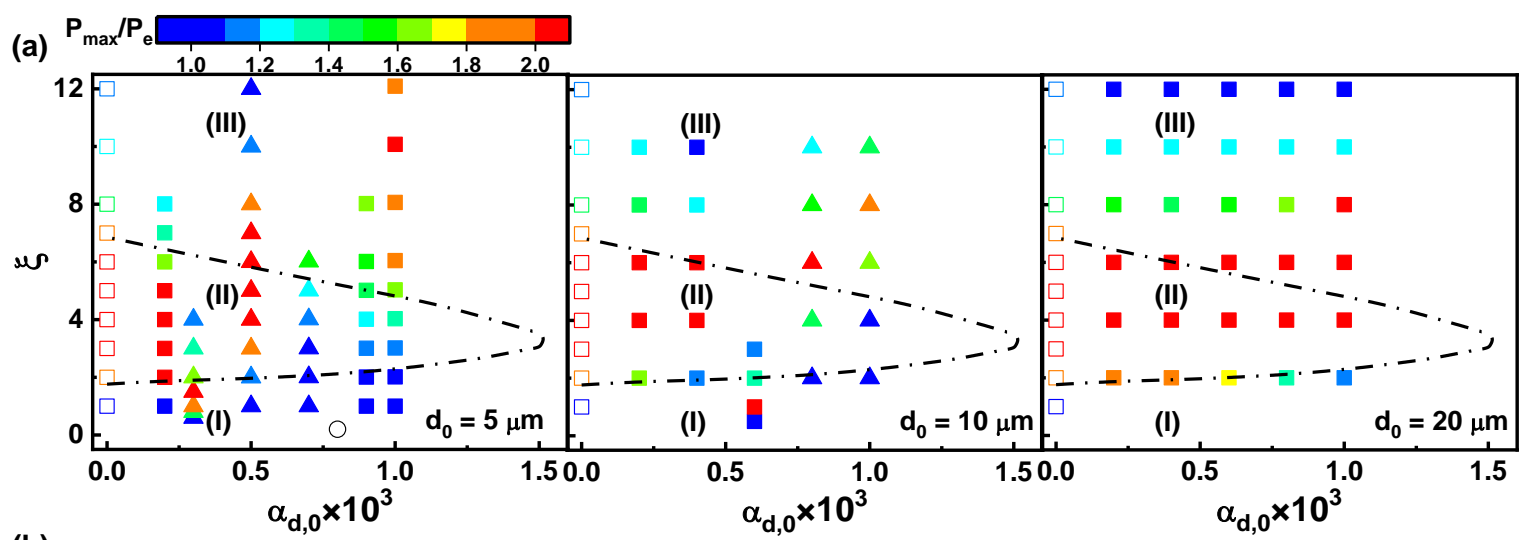

(b)

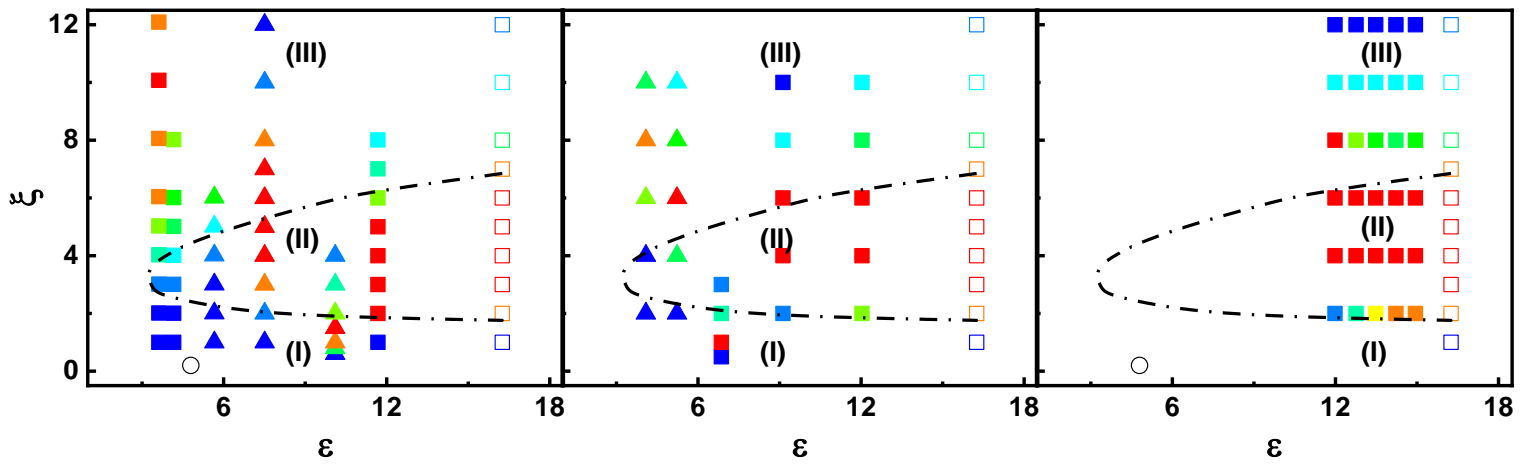

Figure 4. Autoignition mode of stoichiometric $n-\mathrm{C}_{7} \mathrm{H}_{16} /$ air mixtures with water droplets in (a) $\xi-\alpha_{d, 0}$ and (b) $\xi-\varepsilon$ space. Ignition spot size is $r_{0}=3.5 \mathrm{~mm} . d_{0}=5,10$ and $20 \mu \mathrm{m}$ and $T_{0}=1,000 \mathrm{~K}$. Hollow squares denote gaseous 
cases initialized by hot spot, solid squares denote two phase cases initialized by hot spot, solid triangles denote two phase cases initialized by cold spot, solid dash lines are detonation limits abstracted from gaseous cases.

The 1D autoignition modes (i.e., detonation peninsulas) of stoichiometric $n-\mathrm{C}_{7} \mathrm{H}_{16}$ /air mixtures with water droplets due to temperature gradient in an ignition spot are shown in Figure 4. The ignition spot radius is $r_{0}=3.5 \mathrm{~mm}$. The studied cases are summarized in the $\xi-\alpha_{d, 0}$ and $\xi-$ $\varepsilon$ space. A higher $\alpha_{d, 0}$ corresponds to a lower $\varepsilon$. It is seen that the autoignition waves from the ignition spot of water droplet free mixtures (i.e., hollow squares at $\alpha_{d}=0.0$ in each figure) can fall into three categories along $\xi$ : (I) supersonic deflagrative wave, (II) detonative wave with high maximum pressure $\left(P_{\max } / P_{e}\right.$, with $P_{\max }$ and $P_{e}$ being the maximum pressure from 1D calculations and equilibrium pressure from $0 D$ constant-volume autoignition calculations. This normalization can exclude the effect of total heat release [13]) and (III) subsonic deflagrative wave. In this study, we pick up detonative wave modes on condition that $P_{\max } / P_{e} \geq 2$. The detonation limits of water droplet free mixtures diluted by $\mathrm{H}_{2} \mathrm{O}$ vapour are also plotted here. The mole fraction of $\mathrm{H}_{2} \mathrm{O}$ is converted into the equivalent volume fraction. The corresponding results can be found in our previous work [17]. Note that the autoignition wave is typically formed within the ignition spot and travels from ignition spot to outside (from left to right in this study).

The above conventional classification is also suitable for droplet laden mixtures, but special regimes are observed, practically for $5 \mu \mathrm{m}$. It is seen from Figure 4 that, for the finest twophase mixtures, autoignition wave propagates from right to left when $\alpha_{d}=8.0 \times 10^{-4}$ (i.e., hollow circles) due to the corresponding initial gas temperature outside the ignition spot is almost equal to lower turnover temperature. Besides, two opposite autoignition waves are formed for larger $\xi$ when $\alpha_{d}=7.0 \times 10^{-4}$ and $9.0 \times 10^{-4}$ due to the effect of NTC. These cases are not printed in Figure 4. When $\alpha_{d}=1.0 \times 10^{-3}$, the autoignition wave formed within ignition spot is hard to become a detonation wave.

\title{
Conclusions
}

The effects of water droplets on autoignition and detonation development induced by ignition spot with thermal inhomogeneity in $n-\mathrm{C}_{7} \mathrm{H}_{16}$ /air mixture are numerically studied in this work. $\mathrm{A}$ series of droplet diameters and volume fractions are considered. Zero-dimensional two-phase homogeneous ignition under constant-volume condition are first calculated. It is seen that $\tau_{i g}$ of $5 \mu \mathrm{m}$ shows non-monotonic change with $\alpha_{d, 0}$, indicating the NTC behaviours due to the water droplet addition. However, for 10 and $20 \mu \mathrm{m}$, increased $\alpha_{d, 0}$ leads to monotonically increased $\tau_{i g}$. Autoignition modes from an ignition spot in two-phase water-droplet laden $n$ $\mathrm{C}_{7} \mathrm{H}_{16}$ /air mixtures are then analysed based on one-dimensional simulations. It is found that conventional classification is also somewhat suitable for droplet laden mixtures, however special regimes are observed, practically for $5 \mu \mathrm{m}$.

\section{Acknowledgments}

This work used the ASPIRE 1 Cluster from National Supercomputing Centre, Singapore (https://www.nscc.sg/). ZY is supported by the NUS Research Scholarship Budget (Grant Nos. C-261-000-207-532 and C-261-000-005-001).

\author{
Nomenclature \\ d droplet diameter [m] \\ $u_{d} \quad$ droplet velocity [ $\mathrm{m} \mathrm{s}^{-1}$ ] \\ $T_{d} \quad$ droplet temperature [K]
}




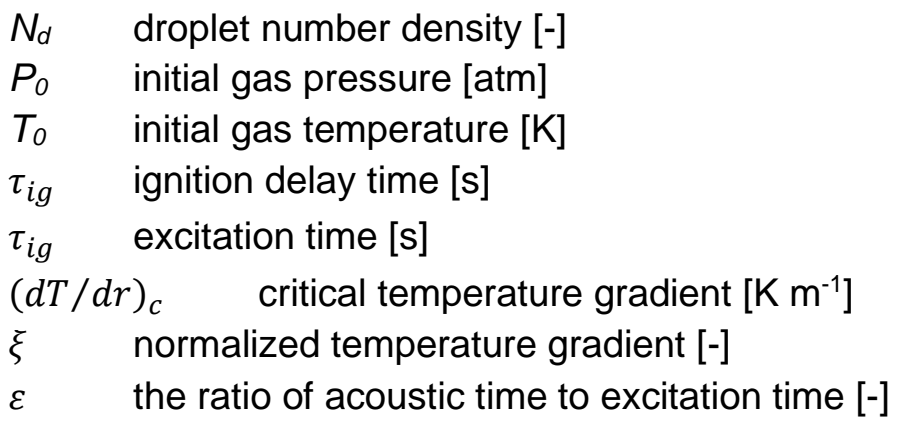

\section{References}

[1] Wang, Z., Liu, H., and Reitz, R. D., 2017, Progress in Energy and Combustion Science, 61, pp. 78112.

[2] Wang, Z., Liu, H., Song, T., Qi, Y., He, X., Shuai, S., and Wang, J., 2015, International Journal of Engine Research, 16(2), pp. 166-180.

[3] Wang, Z., Qi, Y., He, X., Wang, J., Shuai, S., and Law, C. K., 2015, Fuel, 144, pp. 222-227.

[4] Zeldovich, Y. B., 1980, Combustion and Flame, 39(2), pp. 211-214.

[5] Bradley, D., Morley, C., Gu, X. J., and Emerson, D. R., 2002, SAE transactions, 111, pp. 2679-2690.

[6] Gu, X. J., Emerson, D. R., and Bradley, D., 2003, Combustion and Flame, 133(1-2), pp. 63-74.

[7] Bradley, D., and Kalghatgi, G. T., 2009, Combustion and Flame, 156(12), pp. 2307-2318.

[8] Bradley, D., 2012, Philosophical Transactions of the Royal Society A: Mathematical, Physical and Engineering Sciences, 370(1960), pp. 689-714.

[9] Dai, P., Chen, Z., Chen, S., and Ju, Y., 2015, Proceedings of the Combustion Institute, 35(3), pp. 3045-3052.

[10] Dai, P., and Chen, Z., 2015, Combustion and Flame, 162(11), pp. 4183-4193.

[11] Dai, P., and Chen, Z., 2019, Proceedings of the Combustion Institute, 37(4), pp. 4813-4820.

[12] Dai, P., Qi, C., and Chen, Z., 2017, Proceedings of the Combustion Institute, 36(3), pp. 3643-3650.

[13] Dai, P., Chen, Z., and Gan, X., 2019, Combustion and Flame, 201, pp. 208-214.

[14] Boeck, L. R., Kink, A., Oezdin, D., Hasslberger, J., and Sattelmayer, T., 2015, International Journal of Hydrogen Energy, 40, pp. 6995--7004.

[15] Song, Y., and Zhang, Q., 2019, Journal of Hazardous Materials, 363, pp. 16-25.

[16] Zhuang, Y., Li, Q., Dai, P., and Zhang, H., 2020, Fuel, 266(January), p. 117077.

[17] Yu, Z., Zhang, H., and Dai, P., 2021, Physics of Fluids, 33(1), p. 17111.

[18] Sanjosé, M., Senoner, J. M., Jaegle, F., Cuenot, B., Moreau, S., and Poinsot, T., 2011, International Journal of Multiphase Flow, 37, pp. 514-529.

[19] Qiao, L., 2012, Combustion and Flame, 159(2), pp. 673-685.

[20] L. Schiller and A. Naumann, 1935, Zeitschrift des Vereins Deutscher Ingenieure, 77, pp. 318-320.

[21] Huang, Z., and Zhang, H., 2020, Physics of Fluids, 32, p. 123315.

[22] Crowe, C. T., Schwarzkopf, J. D., Sommerfeld, M., and Tsuji, Y., 2011, Multiphase Flows with Droplets and Particles, CRC press.

[23] Yu, H., and Chen, Z., 2015, Combustion and Flame, 162(11), pp. 4102-4111.

[24] Pan, J., Wei, H., Shu, G., and Chen, R., 2017, Combustion and Flame, 185, pp. 63-74.

[25] Chen, Z., Burke, M. P., and Ju, Y., 2009, Proceedings of the Combustion Institute, 32(1), pp. 12531260.

[26] Chen, Z., 2010, Combustion and Flame, 157(12), pp. 2267-2276.

[27] Towery, C. A. Z., Poludnenko, A. Y., and Hamlington, P. E., 2020, Combustion and Flame, 213, pp. 172-183. 